Research Article

\title{
Health State Prediction and Performance Evaluation of Belt Conveyor Based on Dynamic Bayesian Network in Underground Mining
}

\author{
Xiangong Li $\mathbb{D}^{1},{ }^{1}$ Yuzhi Zhang, ${ }^{1}$ Yu Li, ${ }^{1}$ Yujie Zhan, ${ }^{2}$ and Lin Yang $\mathbb{D}^{3}$ \\ ${ }^{1}$ School of Mines, China University of Mining and Technology, Xuzhou 221116, China \\ ${ }^{2}$ School of Industrial and Business Management, Xuzhou College of Industrial Technology, Xuzhou 221116, China \\ ${ }^{3}$ School of Business Administration, Nanjing University of Finance and Economics, Nanjing 210023, China
}

Correspondence should be addressed to Xiangong Li; cumtlxg@163.com and Lin Yang; ylnju@126.com

Received 17 December 2020; Accepted 2 January 2021; Published 20 February 2021

Academic Editor: Tangbin Xia

Copyright ( 2021 Xiangong Li et al. This is an open access article distributed under the Creative Commons Attribution License, which permits unrestricted use, distribution, and reproduction in any medium, provided the original work is properly cited.

To deal with the problem of weak prediction and performance evaluation capabilities of traditional prediction and evaluation methods, a method of health state prediction and performance evaluation of belt conveyor based on Dynamic Bayesian Network (DBN) is proposed. First, the belt conveyor sensor monitoring data are preprocessed to obtain the feature data set with labels. At the same time, qualitative and quantitative analyses and interval discretization are carried out from belt conveyor fault-causing elements to construct the DBN network. Then, the sample data are applied to the DBN network for training, and the DBN-based prediction and performance evaluation model is established. Finally, taking the real-time monitoring data of a belt conveyor in an underground mine as an example, a DBN-based belt conveyor health prediction and evaluation model is constructed to evaluate and predict the health of the equipment. The results show that the model could identify different operating conditions and failure modes and further improves the prediction accuracy.

\section{Introduction}

As a key transport equipment in coal production, the belt conveyor is widely used in underground coal mine and main transport roadway on the ground $[1,2]$. The working environment of belt conveyor in underground mines is often complex and hazardous, which cause belt faults such as conveyor deviation, sliding, broken belt, spreader, and reducer shaft. The health status condition of the belt conveyor directly affects the workload of the working face and the life safety of the coal mine site operators [3-5]. Therefore, accurate prediction and performance evaluation of belt conveyor health status is the necessary and prerequisite for health management of critical equipment in coal mines [6-8].

Most techniques used for equipment health state prediction and performance evaluation fall into two main categories $[9,10]$, which are model-based approaches $[11,12]$ and data-driven approaches $[13,14]$. Most model-based approaches require the identification of accurate physical or mathematical models to describe the process of device health state changes. Data-driven model prediction and performance evaluation methods have become an important approach of prediction and performance assessment for complex equipment for its difficulty to determine a specific health state [15-17]. The data-based prediction approaches are mainly based on data fusion and feature extraction of sensor history data of the system or component to obtain a mapping relationship between data and health states [18, 19]. The method is not combined with the a priori knowledge of the device itself and is a more practical method for prediction and evaluation operations based on the existing collected data and mining the implicit correlation information in the data through various analytical processing methods [20-22]. In general, most of the existing studies focus on prediction and assessment under a single health state and failure mode, ignoring a certain extent of influence of environmental and operational conditions [23-25]. In practical engineering applications, traditional prediction and evaluation methods fall short in massive monitoring data, which in turn affects the effectiveness of prediction [26-28]. 
Based on this, a method was proposed for health state prediction and performance evaluation of belt conveyor based on DBN, carries out quantitative evaluation on the health state of belt conveyor, and predicts the change in the health state of the belt conveyor in the future time slice. First, the historical monitoring data and patrol statistical data on the belt conveyor sensor of the coal mine monitoring system are collected, and the health state prediction and performance evaluation indexes of the belt conveyor are mined. The indexes are discretized to reduce the influence of time parameters on the training results. Then, it analyses the process of DBN network learning and reasoning. Finally, the preprocessed data are input to DBN network for training, and a DBN model for health state prediction and performance evaluation of belt conveyor is established. Experiments show that the proposed approach could effectively solve the shortcoming of traditional methods in data processing, and it has strong feasibility and practicability which could improve the equipment performance in engineering application.

\section{Data-Driven DBN Prediction and Performance Evaluation Method}

In this section, the basic principles of DBNs which include basic assumptions, structure learning, parameter learning, inference, and prediction are introduced. Based on this, a DBN prediction and performance evaluation method is proposed.

2.1. Basic Assumptions of DBN. Bayesian network (BN) is a system model at a given time, which is used to model a system in some states of equilibrium [28]. A Bayesian network can be defined as

$$
\mathrm{BN}=(G, \theta),
$$

where $G$ is the directed acyclic graph of the joint probability distribution over node $Z$ and $\theta$ is a parameter in the network, and the joint probability distribution of $Z$ is as follows:

$$
P\left(Z_{1}, Z_{2}, \ldots, Z_{n}\right)=\prod_{i=1}^{n} P\left(Z_{i} \mid P a\left(Z_{i}\right)\right) .
$$

DBN is a dynamic Bayesian network that simulate the effects of changes in the network over time, reflecting changes in the health of the system at different times. To describe this specific process, some assumptions need to be made as follows:

(1) Steady-state assumption: the conditional probability of a network node is the same for all time slices $t$, and the transfer probability of any two neighbouring time slices is the same.

(2) The first-order Markovian hypothesis: the current state depends only on the state of the previous moment, independent of the state of the previous moment, i.e.,

$$
P\left(Z^{t} \mid Z^{0}, Z^{1}, \ldots, Z^{t-1}\right)=P\left(Z^{t} \mid Z^{t-1}\right) .
$$

2.2. Structural Learning of $D B N$. Based on the above assumptions, a DBN can be defined as $\left(B_{0}, B \longrightarrow\right)$, where $B_{0}$ is the joint probability distribution specifying the initial state $Z[0]$ of the variable and $B \longrightarrow$ refers to the transfer probability $P\left(Z^{t} \mid Z^{t-1}\right)$ (which holds for all $t$ ) on variables $Z[0]$ and $Z[1]$.The Bayesian network formula for two neighbouring time slices is shown in the following equation:

$$
P\left(Z_{t} \mid Z_{t-1}\right)=\prod_{i=1}^{N} P\left(Z_{t}^{i} \mid P a\left(Z_{t}^{i}\right)\right),
$$

where $Z_{t}^{i}$ is the value of the $i$-th variable, moment $t$, and $\mathrm{Pa}\left(Z_{t}^{i}\right)$ is the parent of $Z_{t}^{i}$.

The process in the DBN is fixed and the structure is repeated after the second time slice, and the variable $t=2,3, \ldots$, Tin the slice DBN is kept constant, so that the system can be expressed by only two adjacent slices (i.e., the first and second time slice) and a finite number of parameters can be used to simulate the unbounded sequence length. The probability distribution of the time slice sequences obtained by expanding the 2TBN network is shown as follows:

$$
P\left(Z_{1: T}\right)=\prod_{t=1}^{T} \prod_{i=1}^{N} P\left(Z_{t}^{i} \mid P a\left(Z_{t}^{i}\right)\right) .
$$

DBN is often seen as a generalization of other developments in temporal reasoning, such as the Hidden Markov Model (HMM) and the Kalman Filter Model (KFM). These models can be expressed in a compact form and are popular because of their fast learning and rapid inference techniques. A network example of DBN is shown in Figure 1.

In Figure 1, (a) represents the initial distribution $B_{0}$, (b) represents the conditional distribution $B_{\longrightarrow}$, and (c) represents a network segment formed by the initial network and the transfer network.

2.3. Parameter Learning for Dynamic Bayesian Networks. Based on the above DBN structure, it is necessary to learn the DBN network parameters, i.e., the conditional probability table reflecting the strength of the correlation between the network nodes from a large amount of data, including observation probabilities $P\left(Z_{0}\right), P\left(Z_{1}\right)$, and $P\left(Z_{2}\right)$ and transfer probabilities $P Z_{0}^{t+1}\left|Z_{0}^{t}, P Z_{1}^{t+1}\right| Z_{1}^{t}$, and $P Z_{2}^{t+1} \mid Z_{2}^{t}$. The higher the similarity $\log ^{(P(E \mid \theta))}$ between parameter $\theta$ in the conditional probability table in the DBN and the training data set $E$, the more realistic the results of parameter learning will be. In this paper, the method of maximum release probability is chosen to solve the conditional probability table parameter 1 , and the following maximum release probability equation is constructed:

$$
\log ^{(P(E \mid \theta))}=\log \prod_{i=1}^{n} \prod_{j=1}^{q_{i}} \prod_{i=1}^{p_{i}} \theta_{i j k}^{n_{i j k}},
$$




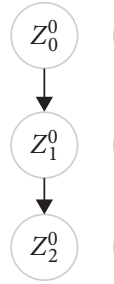

(a)

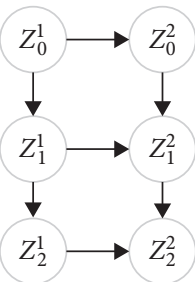

(b)

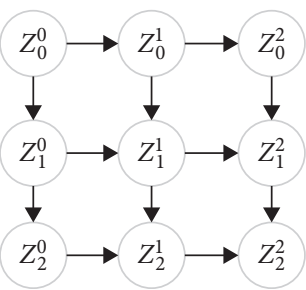

(c)

Figure 1: Example of a dynamic Bayesian network. (a) $B_{0}: P\left(Z_{0}\right)$. (b) $B_{\longrightarrow}: P\left(Z_{1} \mid Z_{2}\right)$. (c) $B_{0} \longrightarrow B_{\longrightarrow}:\left(Z_{0}\right) \longrightarrow P\left(Z_{1} \mid Z_{2}\right)$

where $i$ is all the nodes in the DBN, $j$ is all the parents of $Z_{i}, k$ is the state of $Z_{i}$, and $n_{i j k}$ is the number of samples of the state $k$ of the $i$-th node $Z_{i}$ when its parent is the $j$-th combination:

$$
\begin{cases}\text { Max } & \log ^{P(E \mid \theta)} \\ \text { S.T. } & g_{i j}(\theta)=\sum_{k=1}^{r_{i}} \theta_{i j k}-1=0 .\end{cases}
$$

The process of solving for the maximum probability of likelihood from equation (7) yields $\theta_{i j k}=\left(n_{i j k} / \sum_{k} n_{i j k}\right)$.The sample data for parameter learning are preprocessed historical health status monitoring data, and experiments can be performed by some software such as GeNIe or MATLAB toolbox to complete the DBN parameter learning process.

2.4. DBN Inference Prediction and Evaluation. DBN inferential prediction and evaluation is a key step in the big databased prediction and performance evaluation of DBN belt conveyor health status, and DBN inferential prediction and evaluation is a mathematical method to obtain a posterior distribution by updating the prior distribution of parameter values $\theta$. After determining the DBN structure and parameter learning, it is necessary to analyse each variable in the network and make inferential predictions on the results of specific variables or events. In underground mine transportation system operation, when the belt conveyor health state changes, the monitoring data are entered into the DBN as evidence, and the dynamic Bayesian network could be updated to make inference prediction and evaluation. Finally, through inference calculations, the performance evaluation results of the equipment health state at the observation point at time $t$, and the prediction results of the trend of the belt conveyor health state from time $t$ to time $t+h$ can be obtained.

Assuming that the group of observable nodes $Y=\left[y_{11}, \ldots, y_{1 m}, \ldots, y_{T 1}, \ldots, y_{T m}\right]$ satisfy an independent identically distribution and the group of unknown network nodes are $Z=\left[z_{11}, \ldots, z_{1 n}, \ldots, z_{T 1}, \ldots, z_{T n}\right]$, the dynamic Bayesian network inference rule for $m$ known network nodes when a time slice contains $n$ unknown network nodes is as follows:

$$
P(Z \mid Y)=\sum_{y_{11}, y_{12}, \ldots, y_{T m}} \frac{\prod_{i j} P\left(y_{i j} \mid P a\left(Y_{i j}\right)\right) \prod_{i k} P\left(z_{i k} \mid P a\left(Z_{i k}\right)\right) \prod_{i j} P\left(Y_{i j m}=y_{i j}\right)}{\sum_{z_{11}, \ldots, z_{1 n}, \ldots, z_{T 1}, \ldots, z_{T n}} \prod_{i j} P\left(y_{i j} \mid P a\left(Y_{i j}\right)\right) \prod_{i k} P\left(z_{i k} \mid P a\left(Z_{i k}\right)\right),}
$$

where $P\left(z_{11}, \ldots, \quad z_{1 n}, \ldots, z_{T 1}, \ldots, z_{T n} \mid y_{11}, \ldots, y_{1 m}, \ldots\right.$, $\left.y_{T 1}, \ldots, y_{T m}\right)$ represents the conditional probability density of the variable $z_{11}, \ldots, z_{1 n}, \ldots, z_{T 1}, \ldots, z_{T n}$ with respect to $y_{11}, \ldots, y_{1 m}, \ldots, y_{T 1}, \ldots, y_{T m}, i$ ranges from $i \in[1, T], j$ ranges from $j \in[1, m], k$ ranges from $k \in[1, n], Z_{i k}$ is the value of the unknown network node $z_{i k}, Y_{i j}$ is the value of the observable node $y_{i j}$, and $P a\left(Y_{i j}\right)$ is the parent node of $y_{i j}$.

The performance evaluation process is based on historical monitoring data to evaluate the current condition values, and the inference prediction process is based on historical monitoring data to predict the changes in the health status of the equipment. The DBN performance evaluation and inference prediction formulas are shown in the following equations:

$$
P\left(Z_{t} \mid y_{1: t-1}\right)=\sum_{z_{t-1}} P\left(Z_{t} \mid z_{t-1}\right) P\left(z_{t-1} \mid y_{1: t-1}\right)
$$

where $y_{1: t-1}$ is the evidence value of each condition indicator of belt conveyor health at moments 1 to $t-1$ and $Z_{t}$ is the probability of occurrence of belt conveyor health at moment $t . P\left(Z_{t} \mid y_{1: t-1}\right)$ is the probability of evaluating the occurrence of belt conveyor health at moment $t$ given that the evidence value of each health indicator at moments 1 to $t-1$ is known:

$$
P\left(Z_{t} \mid y_{1: t}\right)=P\left(y_{t} \mid Z_{t}\right)\left[\sum_{z_{t-1}} P\left(Z_{t} \mid z_{t-1}\right) P\left(z_{t-1} \mid y_{1: t-1}\right)\right] \text {, }
$$

where $y_{1: t}$ is the evidence value for each of the health state indicators of the belt conveyor at moment $t$ and $P\left(Z_{t} \mid z_{t-1}\right)$ is the probability of inferentially predicting the occurrence of the health state of the belt conveyor from moment $t$ to moment $t$, given that the evidence value for 
each of the health state indicators from 1 to moment $t$ is known.

\subsection{Data-Driven DBN Prediction and Evaluation Methods.} With the development of Internet of things in underground coal mine, more and more belt conveyor state data could be obtained by different kind sensor on it. Therefore, it is more feasible than ever before to precisely predict the health state and evaluate the performance of belt conveyor. As shown in Figure 2, the steps for data-driven DBN prediction and evaluation are as follows:

Step 1. Collect belt conveyor health status system monitoring data and preprocess to get a labelled feature data set

Step 2. Construct the DBN Topology

Step 3. Dynamic Bayesian network parameter learning

Step 4. Identifying observational evidence, dynamic Bayesian network evaluation, and inferential prediction

For underground mine belt conveyor health state prediction and performance evaluation, the above proposed method is mainly based on machine learning approaches. With the data obtained from the sensor or monitoring system, feature engineering is needed in the early stage where raw data should be processed and the key features should be selected to reduce the dimensionality of the training problem. Based on the DBN model, the performance evaluation and health state prediction could be conducted by inference.

\section{Condition Prediction and Performance Evaluation of DBN Model}

As a key equipment in coal mines, the actual performance of the belt conveyor will decrease over time due to various factors, leading to an increase in the probability of machine failure. To avoid economic losses and casualties due to sudden belt conveyor failure, it is necessary to provide enough time for maintenance, make a reasonable assessment of its current operating state, and make reasonable predictions of future changes in its operating state. A datadriven DBN belt conveyor health status prediction and performance evaluation method are proposed by combining real-time big data of belt conveyor health monitoring system and the advantages of deep learning. The flowchart of the method is shown in Figure 3, after which the specific steps model application are as follows:

(1) DBN network model construction: first, the belt conveyor health status historical data are processed, and the data are discretized to obtain the belt conveyor health status samples. Then, an initial DBN network model is constructed by using the experience of experts, and the training set data are parameterized to obtain a data-driven DBN belt conveyor health status prediction and performance evaluation model.

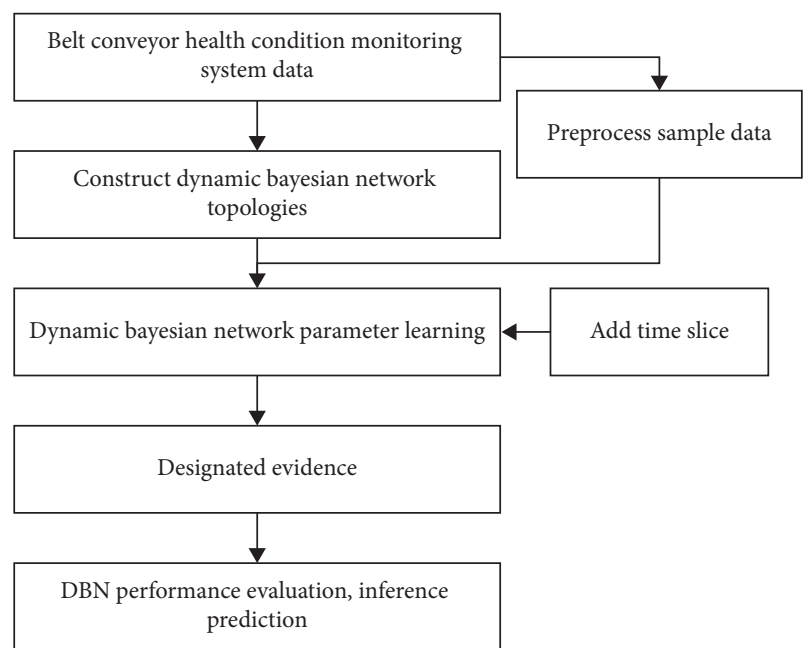

Figure 2: Dynamic bayesian network modeling flowchart.

(2) Belt conveyor health status prediction and performance evaluation: the values of belt conveyor health status parameters obtained from the belt conveyor health status monitoring system are entered into the DBN network model as evidence, and the DBN model is used to evaluate future belt conveyor health status, predict future belt conveyor health status changes, and propose safety maintenance decisions.

\section{Case Study}

4.1. Data Sources. The data used in this case are all from the actual data collected on-site by a new system of Internet of Things (IOT) in a coal mine in Shanxi province. From 2012, the coal mining company carried out a national demonstration project of the Internet of Things and introduced a new system of mine Internet of Things consisting of sensors, cloud platforms, and software systems. The enterprise has achieved certain results in data analysis after the development of two stages of the perceptual mine and the current development of intelligent mines. The big data provided by the mine IOTs are highly accessible and can realize real-time automatic collection of information, high-speed network transmission, standardized integration, three-dimensional visualization simulation, automatic operation, and intelligent decision-making. The real-time monitoring system of belt conveyor operation status can obtain eight types of typical belt conveyor health status, specifically involving belt stacking, belt fumes, belt overload, belt longitudinal tear, belt runaway, belt slip, belt breakage, normal system functions as shown in Figure 4. In this paper, the health status of a belt conveyor located in the main transport belt system of the mine's lean production platform is selected for the prediction and performance evaluation study.

The health status of a belt conveyor can be represented by a series of parameters that reflect its status. There are two ways to obtain health parameters, one is through the sensor in real time, and the other is from the periodic inspection statistics. The real-time monitoring data obtained from the sensors in the monitoring system include characteristic 


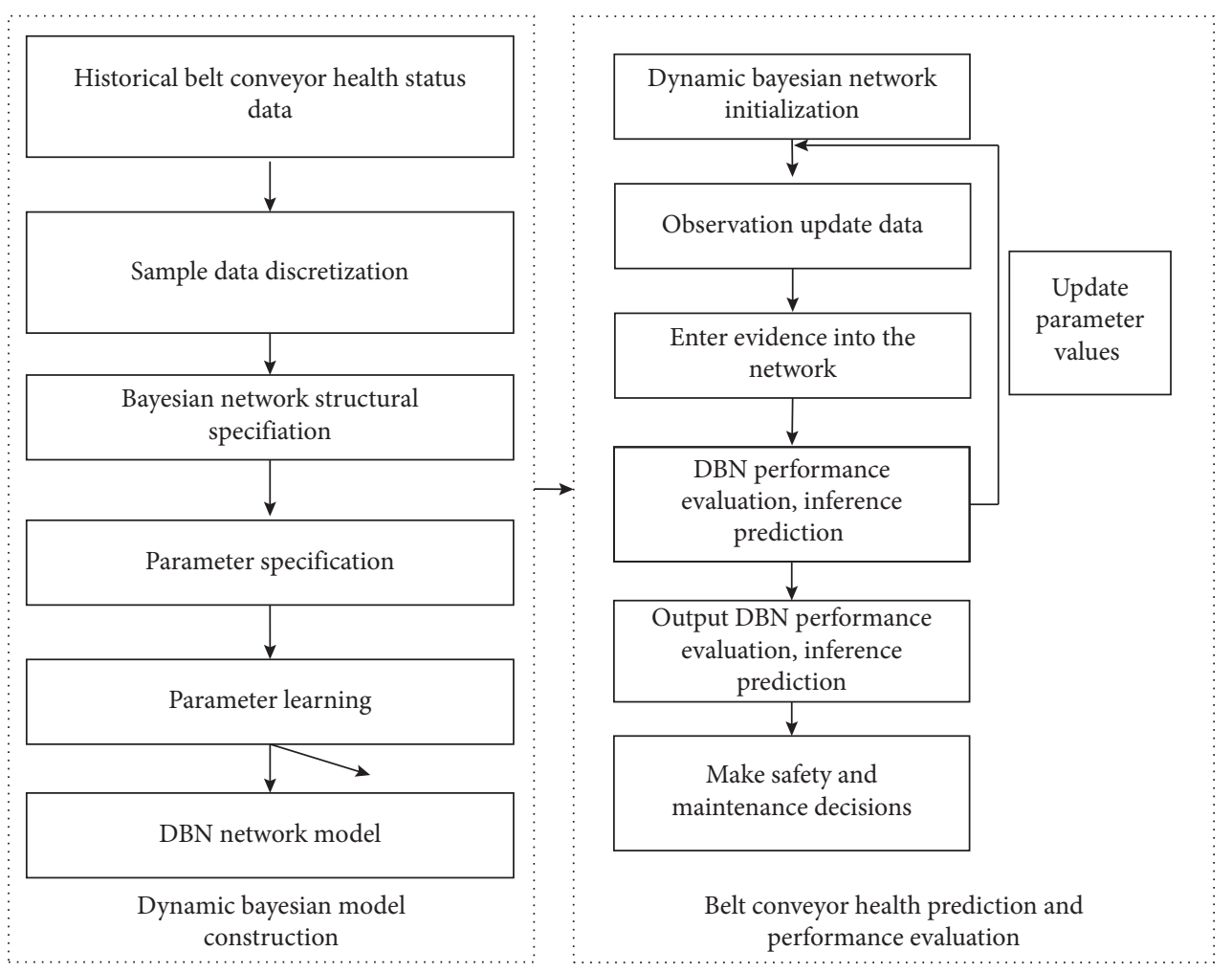

Figure 3: Mode application flow chart.

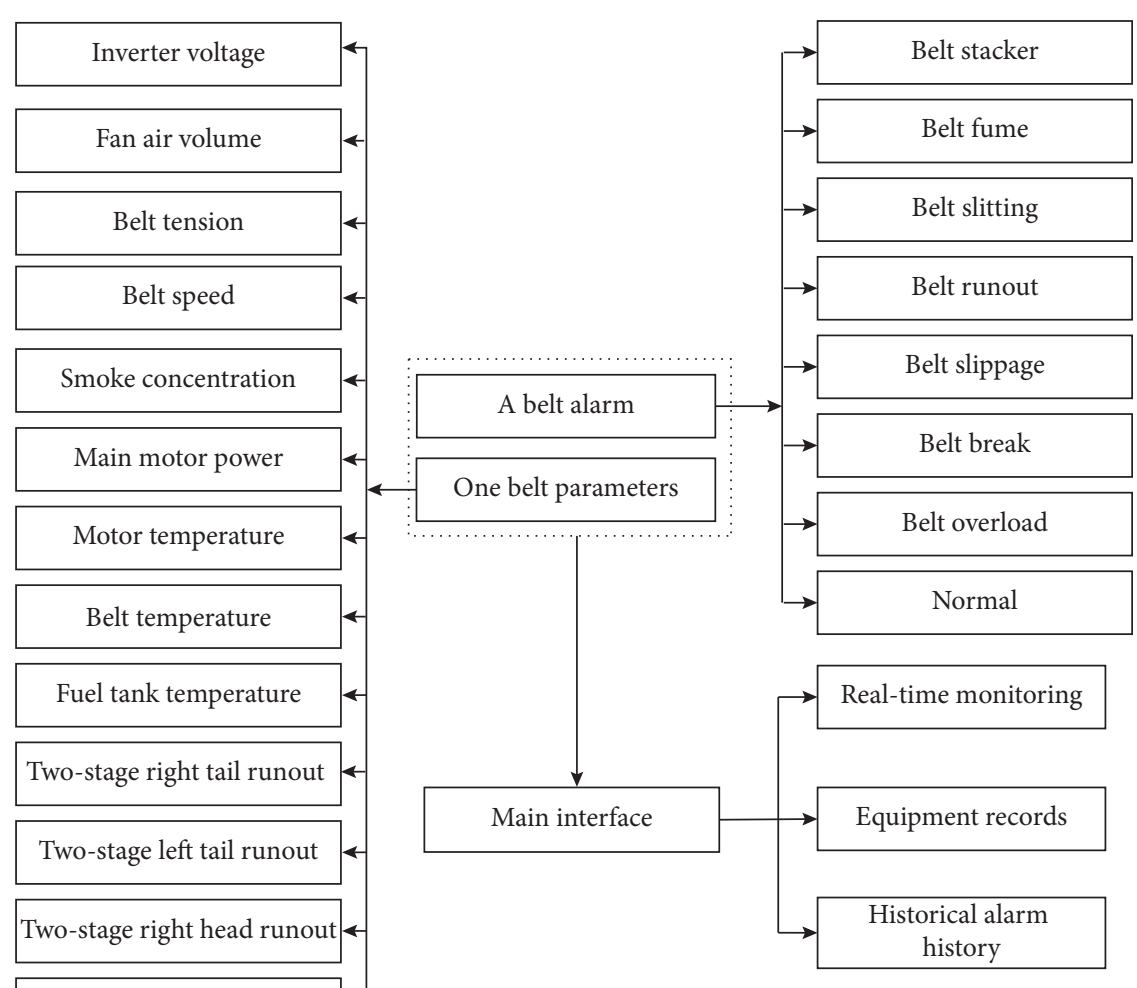

Two-stage head runout

FIgURE 4: Belt conveyor health condition monitoring system functions. 
parameters that can accurately reflect the operating status of the equipment and are easy to measure, specifically power, temperature, speed, offset, smoke concentration, conveyor belt tension, vibration, voltage, current, flow pressure, etc. The health parameters can be obtained from the sensors in real time, and the health parameters have different timeliness in predicting the health status. In this paper, only the health parameters collected by the coal mine belt conveyor operating status real-time monitoring system over a period are selected for analysis, and the sample data are shown in Table 1.

The above characteristic parameters constitute the set of variables of the dynamic Bayesian network and are denoted as $Z=\left\{Z_{1}, Z_{2}, \ldots, Z_{14}\right\}$, where $Z_{1} \sim Z_{13}$ represents main motor power, motor temperature, belt temperature, fuel tank temperature, two-stage right tail runout, two-stage left tail runout, two-stage right head runout, two-stage head runout, smoke concentration, belt speed, belt tension, and fan air volume, inverter voltage, and $Z_{14}$ is the belt conveyor health, respectively. Status levels, corresponding to discretization values, represent the belt conveyor health status of very good, good, fair, poor, and very poor. Since the values of some of the characterization parameters are continuous, the variables are discretized as discontinuous. Based on expert experience and the statistical eigenvalues of the different variables, the continuous intervals are divided to obtain the discretization results shown in Table 2, where the corresponding discretization values are the risk level of each variable.

Due to the lack of sample data on the health status of belt conveyors, this paper collects the abnormal operation data from belt maintenance personnel and compiles the sample data containing abnormal operation data and real-time monitoring data of the normal operation of the system. The sampling frequency of the system real-time monitoring data was recorded every 10 seconds, and the recording frequency of manual statistical data was recorded once a week. The 17280 historical monitoring data within 48 hours were taken according to the time series, the time step of the dynamic Bayesian network was set to 2, and the first 17200 data and the last 80 data were selected as the training set of the model, respectively.

\subsection{Belt Health Prediction and Performance Evaluation Model for Main Belt Conveyors}

4.2.1. Determination of the DBN Structure and Parameter. After identifying the nodes of a dynamic Bayesian network and determining the node values, it is necessary to construct the relationships between the nodes. In this paper, the expert knowledge method is chosen to construct the node relationships, and the set of relationships is established sequentially as follows:

$$
A=\left\{Z_{14} \longrightarrow Z_{i}\right\}
$$

where $i$ takes the range of $i \in(1,2,3, \ldots, 13)$. The initial dynamic Bayesian network through the set of relations is shown in Figure 5.
After the initial dynamic Bayesian network structure is determined, the DBN parameters are learned from a sample of belt health data from the main conveyor section of the belt. The learning process is dynamic and the learned DBN parameters become more and more accurate as the operating time of the belt conveyor changes.

4.2.2. DBN Prediction and Performance Evaluation. The evidence and posterior probability distributions in the DBN network model are distributed according to time, and this paper selects the test set data in the data set to verify the contribution of the model in belt conveyor health status prediction and performance evaluation. First, we set up the time evidence and divide the test set of belt conveyor health monitoring data into 40 test set groups, and the eigenvalues at the moment $t=0$ of each group are entered into the belt health prediction model to evaluate the performance of belt conveyor health in the first ten seconds and predict the belt conveyor health at the moment $t=1$ as evidence. The evidence data of test set 1 is shown in Table 3 .

Taking the test set 1 as an example, the belt conveyor health status data at $t=0$ is input into the model as evidence assignment, and the belt conveyor health status evaluation at $t=0$ and the belt conveyor health status prediction at $t=1$ can be obtained, respectively, as shown in Figure 6. The probability distribution of each network node can be clearly seen from the bar graph of each node in the figure, and each health state has a different conditional probability table at different network nodes. The probability of belt conveyor health condition at $t=0$ and $t=1$ changes, and the probability of belt coal stacking increases significantly, and belt safety managers should take corresponding measures to manage the belt coal stacking phenomenon. Comparing the prediction and performance evaluation results of test set 1 with the actual situation, it can be found that there is inconsistency between the prediction data in test set 1 and the actual data, in which the prediction result of node X11 deviates greatly from the actual. The historical data of belt conveyor health condition and belt tension of belt conveyor has been in a relatively safe range for the past seven days, so it is judged that the excessive belt tension on that day is an emergency, while other inconsistent prediction results are in a small error range. The model is more accurate for belt conveyor health condition prediction and performance evaluation, which meets the basic requirements of actual belt conveyor health condition prediction and performance evaluation.

Set the experimental step to 10 to get the trend of the health state of the belt conveyor, as shown in Figure 7.

In Figure 7, "BCS”, “BS", "LTFB", "BD”, and "BSL", "BB", "BO", and " $N$ " represent the eight types of belt conveyor health, respectively. "BCS" is the belt stacker, "BS" is the belt fume, "LTFB" is the belt slitting, "BD" for the belt runout, "BSL" for the belt slip, "BB" for the belt breakage, and "BO" for the belt overload. "N" is the normal condition. The eight belt conveyor health states have changed over time, with the probability of a belt coal stacking condition increasing significantly. At this point, the belt safety manager 
TABLE 1: Health characteristics of coal mine belt conveyors in operating condition.

\begin{tabular}{|c|c|c|c|c|c|c|c|c|}
\hline Characterization & Belt stacker & Belt fume & Belt slitting & Belt runout & Belt slippage & Belt break & Belt overload & Normal \\
\hline Main motor Power (KW) & 345.23 & 345.65 & 284.51 & 283.29 & 206.45 & 286.9 & 348.25 & 248.55 \\
\hline Motor temperature $\left({ }^{\circ} \mathrm{C}\right)$ & 35.2 & 34.6 & 36.7 & 34.8 & 82.1 & 86.5 & 36.3 & 34.9 \\
\hline Belt temperature $\left({ }^{\circ} \mathrm{C}\right)$ & 55.8 & 55.5 & 56.5 & 55.7 & 88.3 & 86.5 & 55.6 & 55.6 \\
\hline Fuel tank temperature $\left({ }^{\circ} \mathrm{C}\right)$ & 56.4 & 55.6 & 56.2 & 56.1 & 55.9 & 58.9 & 56.3 & 55.9 \\
\hline Two-stage right tail runout $(\mathrm{cm})$ & 7 & 0 & -53 & 5 & 0 & -3 & 0 & 0 \\
\hline Two-stage left tail runout $(\mathrm{cm})$ & -7 & 0 & 53 & -5 & 0 & 3 & 0 & 0 \\
\hline Two-stage right head runout $(\mathrm{cm})$ & 0 & 0 & -5 & 24 & 0 & -63 & 13 & 0 \\
\hline Two-stage head runout $(\mathrm{cm})$ & 0 & 0 & 5 & -24 & 0 & 63 & -13 & 0 \\
\hline Smoke concentration $(\mathrm{mg} \cdot \mathrm{m}-2)$ & 0.26 & 0.52 & 0.13 & 0.14 & 0.13 & 0.16 & 0.19 & 0.14 \\
\hline Belt speed $(\mathrm{m} \bullet \mathrm{s}-1)$ & 2.45 & 3.51 & 2.55 & 3.53 & 3.51 & 0 & 2.62 & 3.51 \\
\hline Belt tension $(\mathrm{kg})$ & 82.3 & 82.1 & 86.5 & 87.4 & 77.1 & 89.6 & 92.4 & 80.0 \\
\hline Fan air volume $(\mathrm{m} \bullet \mathrm{s}-1)$ & 6.15 & 6.19 & 6.13 & 6.12 & 6.13 & 6.15 & 6.15 & 6.14 \\
\hline Inverter voltage $(\mathrm{v})$ & 20.5 & 20.1 & 19.6 & 19.5 & 19.4 & 19.6 & 20.5 & 19.4 \\
\hline
\end{tabular}

TABLE 2: Variable interval discretization classification.

\begin{tabular}{lcc}
\hline Variables & Variable intervals & Corresponding discretized values \\
\hline Main motor power $(\mathrm{KW})$ & {$[0,248],[248,-]$} & 1,2 \\
Motor temperature $\left({ }^{\circ} \mathrm{C}\right)$ & {$[0,70],[70,75],[75,80],[80,-]$} & $1,2,3,4$ \\
Belt temperature $\left({ }^{\circ} \mathrm{C}\right)$ & {$[0,55],[55,85],[85,90],[90,-]$} & $1,2,3,4$ \\
Fuel tank temperature $\left({ }^{\circ} \mathrm{C}\right)$ & {$[0,85],[85,90],[90,95],[95,-]$} & $1,2,3,4$ \\
Two-stage right tail runout $(\mathrm{cm})$ & 0 & 1,2 \\
Two-stage left tail runout $(\mathrm{cm})$ & {$[1],[0]$} & 1,2 \\
Two-stage right head runout $(\mathrm{cm})$ & {$[1],[0]$} & 1,2 \\
Two-stage head runout $(\mathrm{cm})$ & {$[1],[0]$} & 1,2 \\
Smoke concentration $\left(\mathrm{mg} \cdot \mathrm{m}^{-2}\right)$ & {$[1],[0]$} & 1,2 \\
Belt speed $\left(\mathrm{m} \cdot \mathrm{s}^{-} 1\right)$ & {$[0,3.15],[3.15,3.5],[3.5,4],[4,-]$} & $1,2,3,4$ \\
Belt tension $(\mathrm{kg})$ & {$[0.25,6],[6,8,8,10,10,15]$} & 1,2 \\
Fan air volume $\left(\mathrm{m} \cdot \mathrm{s}^{-} 1\right)$ & {$[0,20],[20,25],[25,-]$} & $1,2,3,4$ \\
Inverter voltage $(\mathrm{v})$ & {$[0,0.2],[0.2,0.4],[0.4,0.6][0.6,0.8],[0.8,1]$} & $1,2,3$ \\
Health status levels & & $1,2,3,4,5$ \\
\hline
\end{tabular}

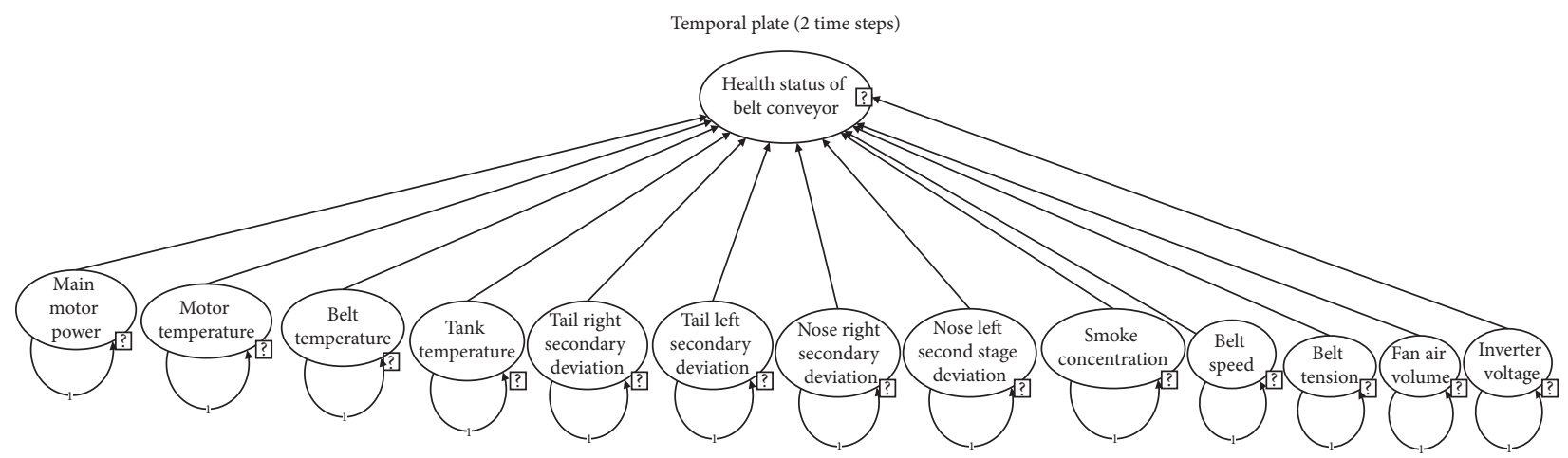

Figure 5: Initial dynamic bayesian network model.

is required to deal with the failure condition in a timely manner to prevent more serious damage.

4.3. Results and Discussion. With the development of IOT application in underground mine, more and more data are obtained. Meanwhile, more machine learning method could be used to fill the gap between data and useful information. To apply a dynamic Bayesian network-based model for equipment health prediction and performance evaluation, there are 2 issues need to be addressed:

(1) Compared with other prediction methods, DBN construction can combine both expert knowledge and machine equipment operating condition data to reduce model construction difficulty and use probability distribution tables and graphical structure to represent the uncertainty relationship between 
Table 3: Test set 1 node evidence data table.

\begin{tabular}{|c|c|c|c|c|}
\hline $\begin{array}{l}\text { Characterization } \\
\text { parameters }\end{array}$ & $\begin{array}{l}t=0 \text { moment } \\
\text { eigenvalues }\end{array}$ & $\begin{array}{c}t=0 \text { moment corresponds to the } \\
\text { value of the discretization }\end{array}$ & $\begin{array}{c}t=1 \text { moment } \\
\text { eigenvalue }\end{array}$ & $\begin{array}{c}t=0 \text { moment corresponds to the } \\
\text { value of the discretization }\end{array}$ \\
\hline Main motor Power $(\mathrm{kW})$ & 283.29 & 2 & 345.23 & 2 \\
\hline Motor temperature $\left({ }^{\circ} \mathrm{C}\right)$ & 34.8 & 1 & 35.2 & 1 \\
\hline Belt temperature $\left({ }^{\circ} \mathrm{C}\right)$ & 55.7 & 2 & 55.8 & 2 \\
\hline $\begin{array}{l}\text { Fuel tank temperature } \\
\left({ }^{\circ} \mathrm{C}\right)\end{array}$ & 56.1 & 1 & 56.4 & 1 \\
\hline $\begin{array}{l}\text { Two-stage right tail } \\
\text { runout }(\mathrm{cm})\end{array}$ & 0 & 2 & 0 & 2 \\
\hline $\begin{array}{l}\text { Two-stage left tail runout } \\
(\mathrm{cm})\end{array}$ & 0 & 2 & 0 & 2 \\
\hline $\begin{array}{l}\text { Two-stage right head } \\
\text { runout }(\mathrm{cm})\end{array}$ & 1 & 1 & 0 & 2 \\
\hline $\begin{array}{l}\text { Two-stage head runout } \\
(\mathrm{cm})\end{array}$ & 0 & 2 & 0 & 2 \\
\hline $\begin{array}{l}\text { Smoke concentration } \\
\left.\left(\mathrm{mg} \cdot \mathrm{m} . \mathrm{s}^{-1}\right)\right)\end{array}$ & 0 & 2 & 0 & 2 \\
\hline Belt speed $\left(\mathrm{m} \bullet \mathrm{s}^{-1}\right)$ & 3.53 & 2 & 2.46 & 2 \\
\hline Belt tension (kg) & 87.5 & 2 & 104.2 & 2 \\
\hline Fan air volume $\left(\mathrm{m} \bullet \mathrm{s}^{-1}\right)$ & 6.12 & 2 & 6.16 & 2 \\
\hline Inverter voltage $(\mathrm{v})$ & 19.6 & 1 & 20.6 & 1 \\
\hline
\end{tabular}

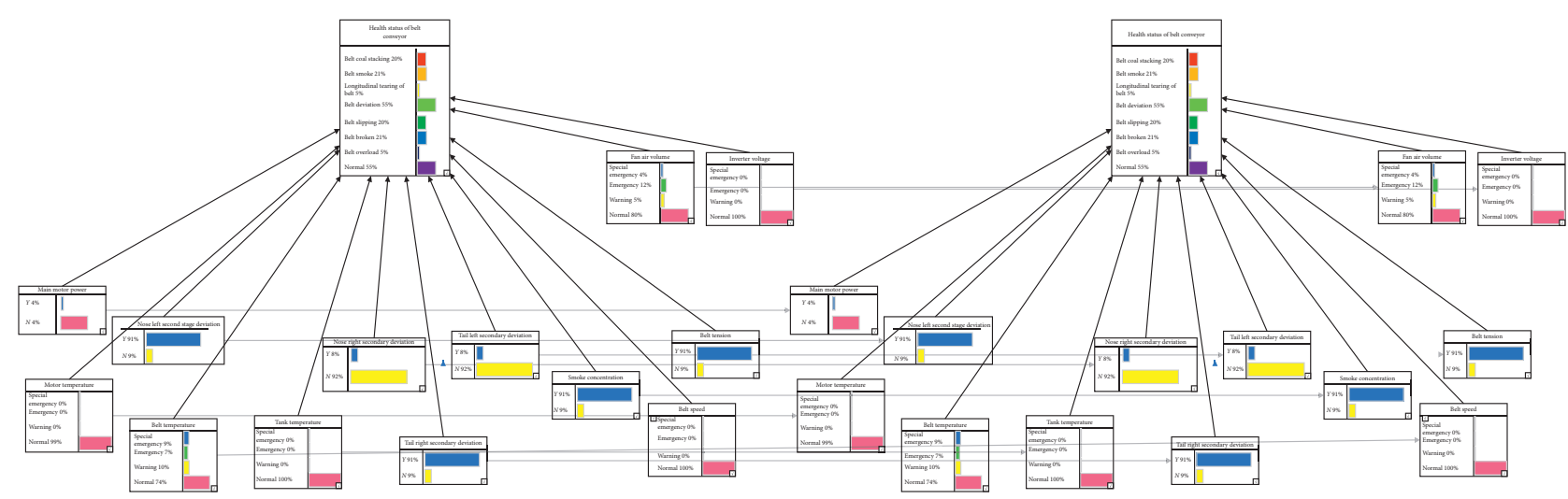

FIGURE 6: DBN prediction and performance evaluation results of belt conveyor.

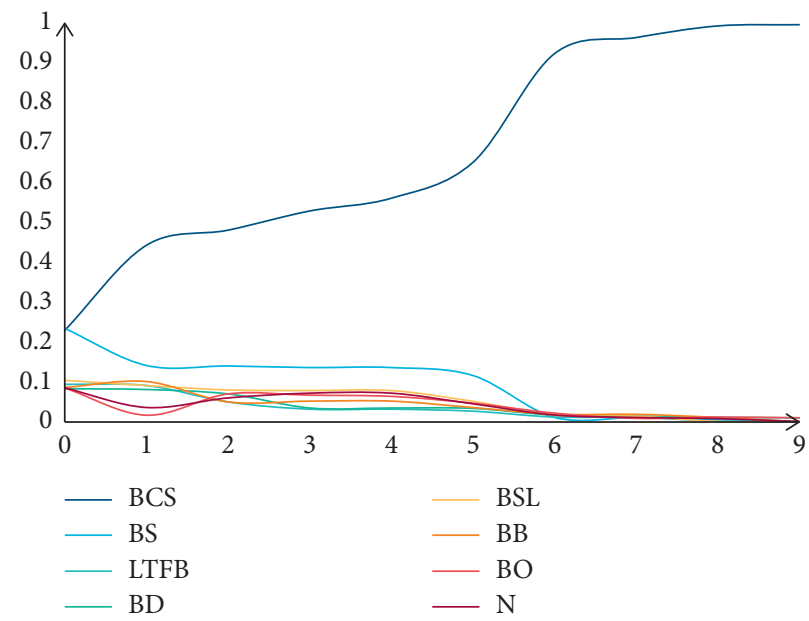

Figure 7: Trend of belt conveyor health status. 
variables in a temporal context, which is basically in line with the need of mining uncertainty relationship between equipment health condition and characteristic parameters. At the same time, the prediction method can update the evidence using real-time updated data from machine equipment, thus reducing errors in prediction and performance evaluation.

(2) Among the 40 test sets, some of them have more than 5 deviations from the prediction, and the prediction effect is not so ideal, while the better-performing test sets have more conformity between the predicted and actual conditions. Analysing these test sets with unsatisfactory prediction results, the following possibilities can be suggested: (1) The possibility of contingency: the possibility of contingency is mentioned in the analysis of the Z11 node in test set 1 . This refers to a situation that has been stable for the current time, but the current state is less likely to occur. A contingency does not mean a situation that should not happen but rather a situation that happens with a small probability. This is a possibility of error because it is a common occurrence in real problems. (2) Changes in influencing factors: since the data used in this paper are for a 48-hour period, the time span of the data is small, and during this period, there may be factors related to the improvement of belt conveyor maintenance technology, etc., which may have some impact on the health status. For example, if there is a defect in the main motor, the indicator is at a high safety level for the first 5 hours, but after the 5th hour, up to the 48th hour, the level of risk is often high, but in general, it has been significantly reduced and the trend of the risk level is stable. Therefore, this is a possible scenario for error generation.

\section{Conclusions}

The fault of belt conveyor in underground mine is analyzed and fault feature are selected from the monitoring Indicators. Based on data preprocessing, a dynamic Bayesian network is constructed after which the parameter is obtained by learning algorithm. It is proved that the proposed method is effective to assess and predicted the health performance of the conveyor belt. The main conclusions are as follows:

(1) The prediction and performance evaluation method proposed in this paper is based on a real-time datadriven dynamic Bayesian network model, and the accuracy of the results is $92 \%$ and $80 \%$ compared with the actual situation. The model could predict and evaluate the health status of belt conveyors and improve the accuracy of prediction and performance evaluation.

(2) Based on the analysis of the results, it is recommended that the data volume of the belt conveyor performance monitoring system should be increased to improve the prediction and warning efficiency of the belt conveyor from the source and avoid accidents caused by unstable belt health status.

(3) The belt conveyor health state prediction and performance evaluation are meaningful to promote the solution of coal mine machinery reliability problems and thus improve the reliability of the coal mine machinery system and ensure the normal implementation of coal mine safety management. In actual production, the mine machinery system is mostly a linked multidevice joint production system. Therefore, the method can be applied to the prediction of equipment health status and performance evaluation of multiple machines.

\section{Data Availability}

The data used to support the study are available from the corresponding author upon request.

\section{Conflicts of Interest}

The authors declare that there are no conflicts of interest regarding the publication of this paper.

\section{Acknowledgments}

This study was supported by the National Key Research and Development Project of China (grant no.: 2017YFC0804408).

\section{References}

[1] C. J. Alonso-Gonzalez, N. Moya, and G. Biswas, "Dynamic Bayesian network factors from possible conflicts for continuous system diagnosis," In Advances in Artificial Intelligence, Springer, Berlin, Germany, 2011.

[2] W. Kawalec, R. Król, and N. Suchorab, "Regenerative belt conveyor versus haul truck-based transport: polish open-pit mines facing sustainable development challenges," Sustainability, vol. 12, no. 21, p. 9215, 2020.

[3] A. K. S. Jardine, D. Lin, and D. Banjevic, "A review on machinery diagnostics and prognostics implementing condition-based maintenance," Mechanical Systems and Signal Processing, vol. 20, no. 7, pp. 1483-1510, 2006.

[4] Y. G. Lei and Z. J. He, "Advances in applications of hybrid intelligent fault diagnosis and prognosis technique," Journal of Vibration and Shock, vol. 30, no. 9, pp. 129-135, 2011.

[5] J. Lee, F. Wu, W. Zhao, M. Ghaffari, L. Liao, and D. Siegel, "Prognostics and health management design for rotary machinery systems-reviews, methodology and applications," Mechanical Systems and Signal Processing, vol. 42, no. 1-2, pp. 314-334, 2014.

[6] M. Ma, C. Sun, and X. Chen, "Deep coupling autoencoder for fault diagnosis with multimodal sensory data," IEEE Trans. Ind. Informat.vol. 14, no. 3, pp. 1137-1145, 2018.

[7] C. Li, R.-V. Sanchez, G. Zurita, M. Cerrada, D. Cabrera, and R. E. Vásquez, "Gearbox fault diagnosis based on deep random forest fusion of acoustic and vibratory signals," $M e$ chanical Systems and Signal Processing, vol. 76-77, pp. 283-293, 2016. 
[8] J. Gai and Y. Hu, "Research on fault diagnosis based on singular value decomposition and fuzzy neural network," Shock and Vibration, vol. 2018, Article ID 8218657, 7 pages.

[9] C. Sun, M. Ma, Z. Zhao, S. Tian, R. Yan, and X. Chen, “Deep transfer learning based on sparse autoencoder for remaining useful life prediction of tool in manufacturing," IEEE Transactions on Industrial Informatics, vol. 15, no. 4, pp. 2416-2425, Apr. 2019.

[10] R. Zhao, R. Yan, Z. Chen, K. Mao, P. Wang, and R. X. Gao, "Deep learning and its applications to machine health monitoring," Mechanical Systems and Signal Processing, vol. 115, pp. 213-237, 2019.

[11] C. F. Sun, Y. R. Wang, Y. Shen et al., "Fault diagnosis of planetary gearbox based on adaptive parameter variational mode decomposition," Journal of Aerospace Power, vol. 33, no. 11, pp. 205-214, 2018.

[12] M. Ma, C. Sun, X. Chen, X. Zhang, and R. Yan, "A deep coupled network for health state assessment of cutting tools based on fusion of multisensory signals," IEEE Transactions on Industrial Informatics, vol. 15, no. 12, pp. 6415-6424, Dec. 2019.

[13] X. Zhang, Z. Liu, Q. Miao, and L. Wang, "An optimized time varying filtering based empirical mode decomposition method with grey wolf optimizer for machinery fault diagnosis," Journal of Sound and Vibration, vol. 418, pp. 55-78, 2018.

[14] Y. G. Lei, F. Jia, X. Zhou et al., "A deep learning-based method for machinery health monitoring with big data," Journal of Mechanical Engineering, vol. 51, no. 21, pp. 55-62, 2015.

[15] Q. Jin, Y. R. Wang, and J. Wang, "Planetary gearbox fault diagnosis based on multiple feature extraction and information fusion combined with deep learning," Journal of Mechanical Engineering, vol. 30, no. 2, pp. 74-82, 2019.

[16] Y. Lei, F. Jia, J. Lin, S. Xing, and S. X. Ding, "An intelligent fault diagnosis method using unsupervised feature learning towards mechanical big data," IEEE Transactions on Industrial Electronics, vol. 63, no. 5, pp. 3137-3147, 2016.

[17] X. Li, W. Zhang, and Q. Ding, "Deep learning-based remaining useful life estimation of bearings using multi-scale feature extraction," Reliability Engineering and System Safety, vol. 182, pp. 208-218, 2019.

[18] J. Gai, J. Shen, H. Wang et al., "A parameter-optimized DBN using GOA and its application in fault diagnosis of gearbox," Shock and Vibration, vol. 2020, p. 11, Article ID 4294095, 2020.

[19] J. Hu, Z. L. Zhang, and Y. Wang, "An intelligent fault diagnosis system for process plant using a functional HAZOP and DBN integrated methodology," Engineering Applications of Artificial Intelligence, vol. 45, pp. 119-135, 2015.

[20] R. Liu, B. Yang, E. Zio, and X. Chen, "Artificial intelligence for fault diagnosis of rotating machinery: a review," Mechanical Systems and Signal Processing, vol. 108, pp. 33-47, 2018.

[21] R. Zhao, R. Yan, J. Wang, and K. Mao, "Learning to monitor machine health with convolutional bi-directional LSTM networks," Sensors, vol. 17, no. 2, 2017.

[22] X. Chen, Z. Shen, Z. He, C. Sun, and Z. Liu, "Remaining life prognostics of rolling bearing based on relative features and multivariable support vector machine," Proceedings of the Institution of Mechanical Engineers, Part C: Journal of Mechanical Engineering Science, vol. 227, no. 12, pp. 2849-2860, 2013.

[23] E. Chemali, P. J. Kollmeyer, M. Preindl, R. Ahmed, and A. Emadi, "Long short-term memory networks for accurate state-of-charge estimation of li-ion batteries," IEEE
Transactions on Industrial Electronics, vol. 65, no. 8, pp. 6730-6739, 2018.

[24] S. Wang, X. Chen, I. W. Selesnick, Y. Guo, C. Tong, and $\mathrm{X}$. Zhang, "Matching synchrosqueezing transform: a useful tool for characterizing signals with fast varying instantaneous frequency and application to machine fault diagnosis," $\mathrm{Me}$ chanical Systems and Signal Processing, vol. 100, pp. 242-288, 2018.

[25] S. Yin, G. Wang, and H. R. Karimi, "Data-driven design of robust fault detection system for wind turbines," Mechatronics, vol. 24, no. 4, pp. 298-306, 2014.

[26] G. W. Vogl, B. A. Weiss, and M. Helu, "A review of diagnostic and prognostic capabilities and best practices for manufacturing," Journal of Intelligent Manufacturing, vol. 6, 2016.

[27] L. Bao, C. Juan, J. Li, and Y. Zhang, "Boosted near-miss undersampling on SVM ensembles for concept detection in large-scale imbalanced datasets," Neurocomputing, vol. 172, pp. 198-206, 2016.

[28] Y. Wu, B. Jiang, N. Lu, and Y. Zhou, "Bayesian network based fault prognosis via Bond graph modeling of high-speed railway traction device," Mathematical Problems in Engineering, vol. 2015, Article ID 321872, 2015. 